Communications in Physics, Vol. 19, No. 1 (2009), pp. 39-44

\title{
TRANSVERSE DISTRIBUTION OF PUMP POWER IN THE DIODE-LASER SIDE-PUMPED SOLID-STATE LASER ROD
}

\author{
MAI VAN LUU, DINH XUAN KHOA, AND VU NGOC SAU \\ Vinh University \\ HO QUANG QUY \\ Academy of Military Science and Technology
}

\begin{abstract}
Based on the assumption that Gaussian pump power of diode laser bar is the same at any cross-section along the laser rod and its curvated surface plays as thin lens, the expression describing the pump intensity distribution inside laser rod was obtained by transfer matrix. To have the cross-section of active volume or excited volume coincides with one of laser mode volume, the dependence of pump intensity distribution on location of outside pump beams is investigated by simulation.
\end{abstract}

\section{INTRODUCTION}

Recently, the diode laser-pumped solid-state lasers from the very small [1] to the kilowatt level of output power $[2,3]$ are interested and developed by because of their efficient use in high technology. Mode size optimization in laser-diode end-pumped lasers has been investigated $[4,5]$. Side-pumping geometry can be used to achieve higher output powers [6]. For analysis in above-mentioned work, there were the following assumptions made: Distribution of diode bar around the rod is assumed to produce an azimuthal uniform illumination; reflection and refraction effects are not considered; to separate the calculation of the absorption profile from how the pump light travels from the diode bar to the surface of the rod, one describes the pump beam from the diodes only after the beams have entered the rod; the pump beam is assumed to travel through the rod only once, i.e. reintroduction of a pump beam through reflectors is not discussed; a single-absorption coefficient can be used to describe the absorption process. Consequently, these assumptions lead to that: first, it is not suitable for optimality of pump stored energy in laser rod; second, one can not choose the optimal parameters for matching between the size of the pump volume and laser mode one; thirdly, it is still not assumed the laser rod as a focusing lens, which is a important fact influences on the pump energy distribution. To advoid above problems, we present a four-side-pumped structure for solid-state laser and a new cross-sectional geometry of the laser rod pumped by diode bar. Pumped by four laser-diode bars, which has a Gaussian distribution in far field, so transverse intensity distribution in active rod of solid-state laser can be changed and influences on dimension of effective "pencil" and then on laser beam structure. 


\section{PUMP INTENSITY DISTRIBUTION}

As shown in Keming's work [7] and Carts's work [8], the cross-sectional geometry of the laser rod pumped by four laser diode bars can be illustrated in Fig. 1. The diode sources are assumed to have a Gaussian emittance profile (transverse distribution) and are conditioned such that they are effectively arrayed uniformly around the rod, i.e. they uniformly distribute along axis-z.

(a)

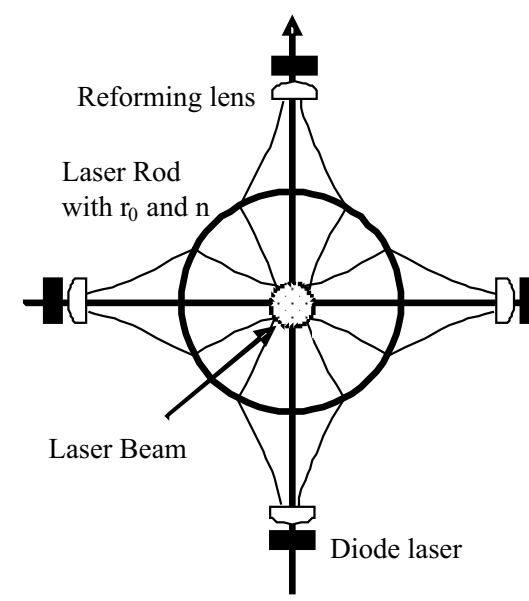

(b)

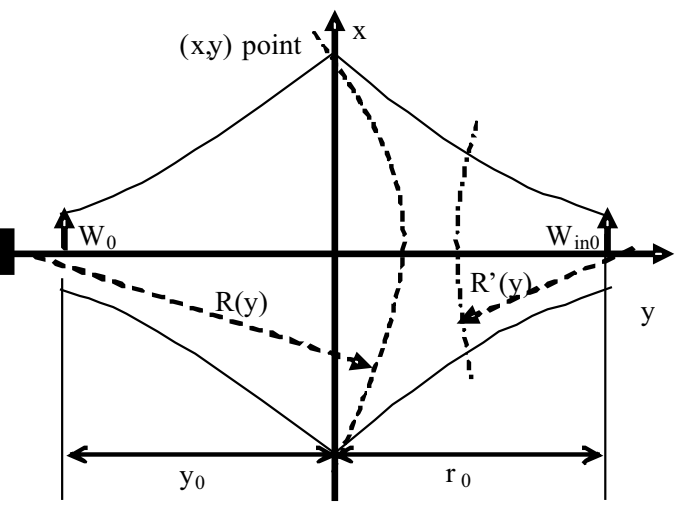

Fig. 1. a- Cavity geometry for four sides-pumping module, b- Cross-sectional geometry of Gaussian beam outside and inside rod

We assume that the laser rod has a radius $r_{0}$ and a refractive index $n$, the Gaussian beam of laser diode bar in cross-section of laser rod, which is placed at point $y_{0}$ from outside surface of rod, has a complex amplitude [9]:

$$
U(x, y)=A_{0}\left[\frac{W_{0}}{W(y)}\right] \exp \left[-\frac{x^{2}}{W^{2}(y)}\right] \exp \left[-j k y-j k \frac{x^{2}}{2 R(y)}+j \xi(y)\right]
$$

where, $y$ is the proprating direction and $x$ is expanding direction,

$$
\begin{aligned}
W(y) & =W_{0}\left[1+\left(\frac{y}{b}\right)^{2}\right]^{1 / 2} \text { is the radius at point y in propagating direction, } \\
R(y) & =y\left[1+\left(\frac{b}{y}\right)^{2}\right] \text { is the wavefront radius of curvature, } \\
W_{0} & =\left(\frac{\lambda b}{\pi}\right)^{1 / 2} \text { is the beam waist, } \\
\xi(y) & =\tan ^{-1}(y / b) \text { is the excess phase (i.e.,initial phase), }
\end{aligned}
$$

and $b$ is the Rayleigh range (see Fig. 1b).

Propagating through the rod from one side, the phase of this beam will be changed as well as after propagating through thin lens (see Fig. 1a) with focal length [9] 


$$
f=r_{0} /(n-1)
$$

so that the complex amplitude transmittance of this lens is proportional toexp $\left(j k x^{2} / 2 f\right)$ and then the phase of the transmitted wave is altered to [9]

$$
k y+k \frac{x^{2}}{2 R(y)}-\xi(y)-k \frac{x^{2}}{2 f}=k y+k \frac{x^{2}}{2 R^{\prime}(y)}-\xi(y)
$$

where

$$
1 / R^{\prime}(y)=1 / R(y)-1 / f
$$

Using (3), (7) and (8), substituting into (1), we obtained the complex amplitude of pump beam inside laser rod, given by

$$
U_{i n}(x, y)=A_{0}\left[\frac{W_{i n 0}}{W_{i n}(y)}\right] \exp \left[-\frac{x^{2}}{W_{i n}^{2}(y)}\right] \exp \left[-j k y-j k \frac{x^{2}}{2 R^{\prime}(y)}+j \xi(y)\right]
$$

where

$$
\left.\begin{array}{l}
W_{i n 0}=M W_{0} ; b_{i n}=M^{2} b ; W_{i n}=W_{i n 0}\left[1+\left(\frac{y}{b_{i n}}\right)^{2}\right]^{1 / 2} ; \\
M=\frac{M_{t}}{\sqrt{1+t}} ; t=\frac{b}{y_{0}-r_{0} /(n-1)} ; M_{t}=\left|\frac{r_{0}}{y_{0}(n-1)-r_{0}}\right| .
\end{array}\right\}
$$

The waist of "inside" beam has the location at center of laser rod when following condition is satisfied

$$
r_{0}^{=} M^{2}\left(y_{0}-\frac{r_{0}}{n-1}\right)+\frac{r_{0}}{n-1}
$$

Using (4), (10) and (11) we obtained the location of waist of "outside" beam

$$
y_{0}=\frac{r_{0}}{n-2}-\frac{\pi W_{0}^{2}}{\lambda}
$$

i.e., it depends on radius and refractive index of laser rod and waist and wavelength of pump beam.

From (9) we have the expression of the pump energy distribution inside the laser rod for single-side-pumping as following

$$
I_{i n}(x, y)=I_{0}\left[\frac{W_{i n 0}}{W_{i n}(y)}\right]^{2} \exp \left[-\frac{2 x^{2}}{W_{i n}^{2}(y)}\right]
$$

We assume that pump intensity distribution (13) is symmetry for center of the rod, which seems to be an origin of co-ordinate system $(x=0, y=0)$, i.e. it is means that

$$
I_{\text {in }}(x, y)=I(-x, y)=I(x,-y)=I(-x,-y) .
$$

Really, every laser diode bar used as a pump lamp having narrow spectra to enhance conversion efficiency. The coherent quality of laser beam is not important for this purpose. Moreover, the absorption of active particles in laser rod to create population inversion is not stimulated, but is spontanuous (every pump photon reaches laser rod at different time after reflection from reflector [10]), so population inversion creating is statistical process and population inversion depends on total pump intensity (the sum of intensities integrated over pulse-duration time of all diode lasers), it means that total excited particles at defined 
time is proportional to sum of intensities of all pump lasers at that time. With considering that the delay time between all lasers is less than lifetime of upper laser level (it means that phase mitmach between all laser beams can be negleted), so that the pump intensity distribution for two opposite sides-pumping is given by

$$
I_{\text {twoside }}(x, y)=I_{\text {in }}(x, y)+I_{\text {in }}(x,-y)
$$

and similarly, for four sides-pumping is given by

$$
I_{\text {fourside }}(x, y)=I_{\text {twoside }}(x, y)+I_{\text {twoside }}(y, x) \text {. }
$$

\section{SIMULATION AND DISCUSSION}

We assume that the parameters of pump beam chosen to be $W_{0}=1 \mathrm{~mm}, \lambda=860 \mathrm{~nm}$ and the parameters of laser rod chosen to be $r_{0}=6 \mathrm{~mm}$, and a refractive index, $n=$ 1.78. The location of waist of pump beam is calculated from (12). Now pump intensity distributions inside the laser rod for side-pumped solid-state laser with two sides and four sides can be obtained as shown in Fig.2a and Fig.2b, respectively.

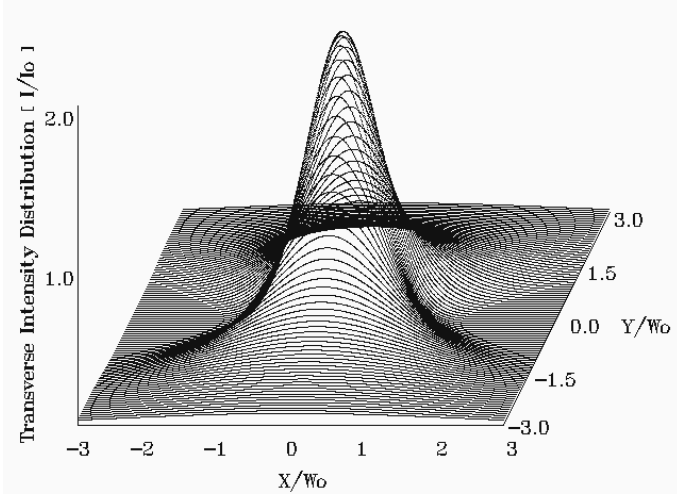

(a)

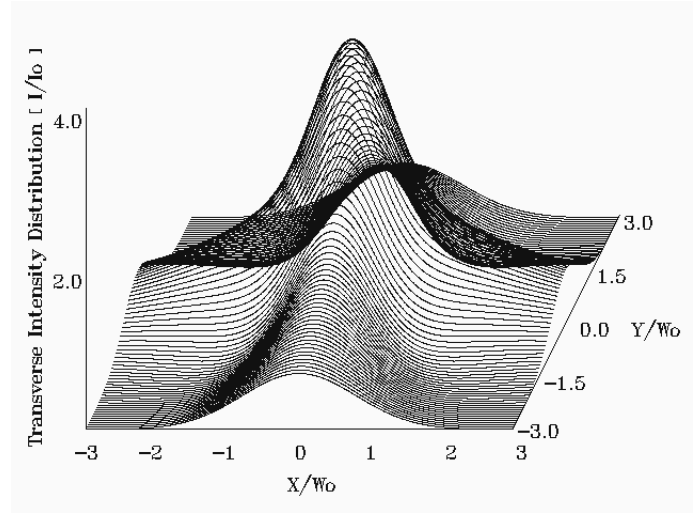

(b)

Fig. 2. Pump intensity distribution for a two-side-pumped (a) and four-sidepumped (b) solid-state lasers

In Fig. 3 can see overlap pump profile in x-axial plane for one-side-pumped (a) and for four side-pumped (b) laser. In Fig. 4 can see overlap pump profile in y-axial plane for one side-pumped (a) and four-side-pumped (b) laser. After comparison between all profiles in two figures (Fig. 3 and Fig. 4), we can conclude that the overlap of the pump intensity distribution at the center of the laser rod closely resembles a Gaussian distribution for the four-side-pumped laser.

The waist $\left(\mathrm{W}_{p}\right)$ of the Gaussian overlap can be changed by changing the location of outside beam. For example, in Fig. 5, one can see that the cross section $\left(\pi \mathrm{W}_{p}^{2}\right)$ at level with the same energy in case of $y_{0}=10 \mathrm{~mm}$ is larger than the one in the case of $y_{0}=15 \mathrm{~mm}$. This means that the effective cross-section defined as cross-section of total intensity distribution at level of $\mathrm{I}_{M A X} / \mathrm{e}$ can be chosen so that it coincides with cross-section of the laser mode 


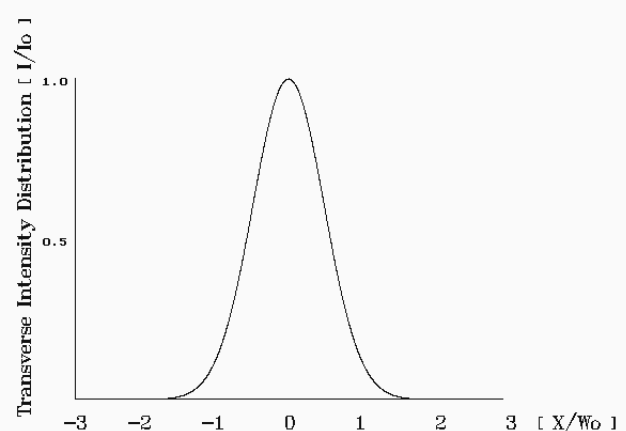

(a)

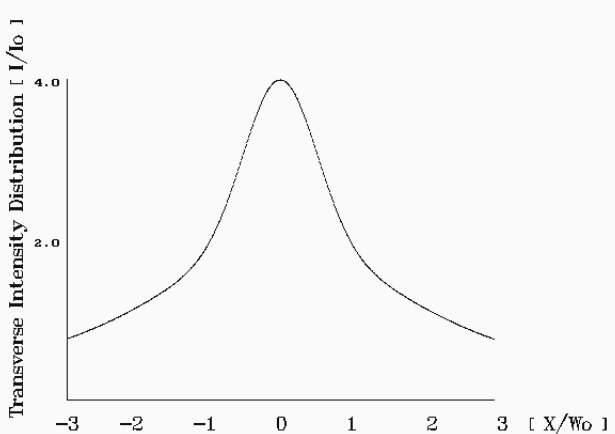

(b)

Fig. 3. Overlap pump-intensity profile in the $\mathrm{x}$-axial plane for one-side-pumped (a) and four-side-pumped (b) lasers

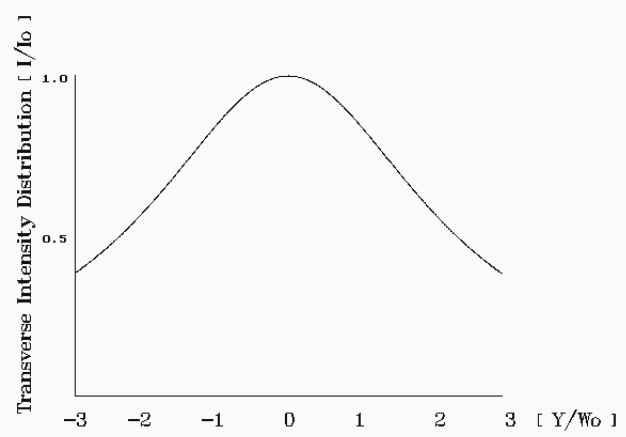

(a)

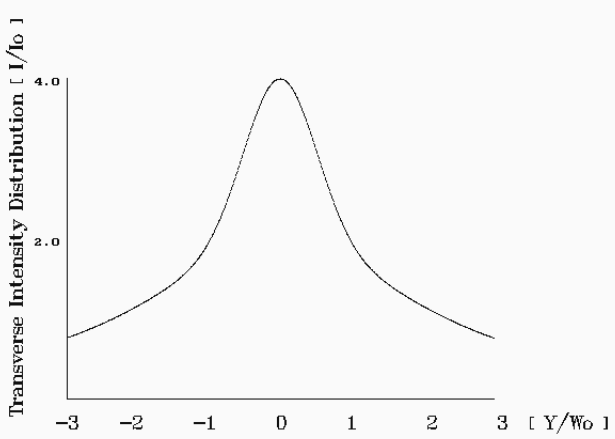

(b)

Fig. 4. Overlap pump-intensity profile in the y-axial plane for one-side-pumped (a) and four-side-pumped (b) lasers

volume $\left(\pi \mathrm{W}_{0 L}^{2}\right)$ by changing the location of outside beam $\left(\mathrm{y}_{0}\right)$ as shown above, when other parameters as beam waist of pump beam $\left(\mathrm{W}_{0 p}\right)$ and pump wavelength $(\lambda)$ are given.

\section{CONCLUSION}

The expression of the pump intensity deposition inside the solid-state laser rod pumped by diode laser bars is introduced. The pump intensity distribution, the pump volume are dependent not only on parameters of pump beam, but also on parameters of laser rod and the location of pump beam from the laser rod. Since that obtained results are useful not only for optimization conversion efficiency, but also for reducing the thermal 


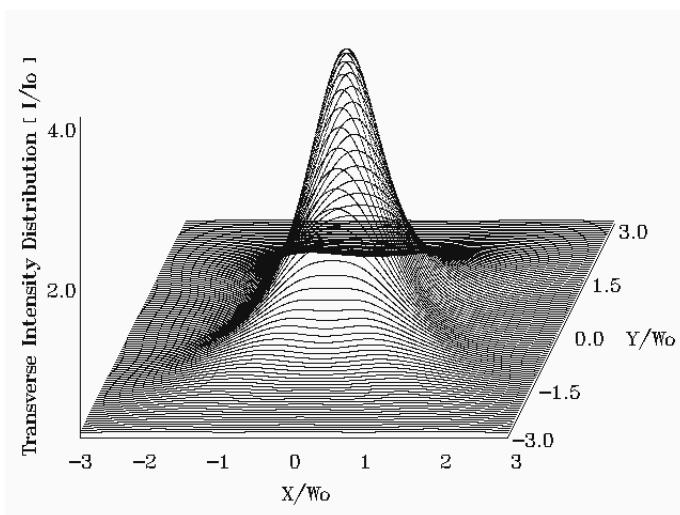

(a)

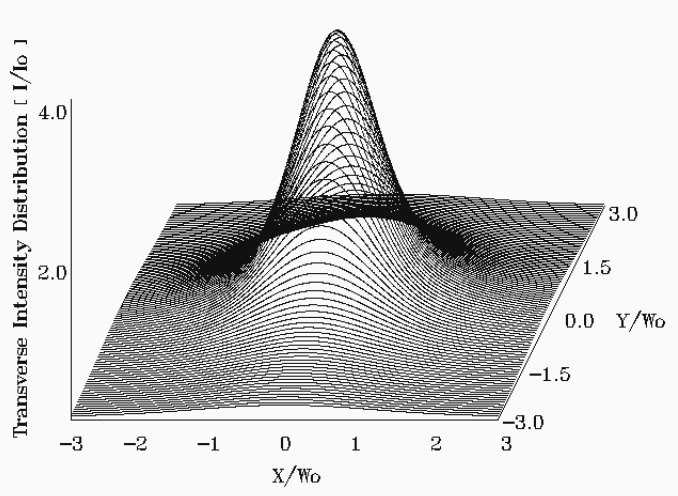

(b)

Fig. 5. Four-side-pump- intensity distribution inside the laser rod with two values of pump beam location: $y_{0}=15 \mathrm{~mm}$ (a) and $y_{0}=10 \mathrm{~mm}$ (b)

effect in laser rod. Moreover, the longitudinal distribution of pump intensity in laser mode volume of the side-pumped laser is important question, which will be investigated in the next article.

\section{REFERENCES}

[1] B. J. Comaskey, et al., IEEE J. Quantum Electron., 28 (1992) 992-996.

[2] N. Hodgson, S. Dong, and Q. Lu, Opt. Lett. 18 (1993) 1727-1729.

[3] R. J. St. Pierre et al., J. Sel.Top. Quantum Electron. 3 (1997) 53-58.

[4] T. Y. Fan, and R. L. Byer, IEEE J. Quantum Electron. 24 (1988) 895-912.

[5] Y. F. Chen et al., IEEE J. Quantum Electron. 33 (1997) 1424-1429.

[6] W. Xie et al., Applied Optics 39 (2000) 5482-5487.

[7] Du Keming et al., Appl. Optics 37 (1998) 2361-2364.

[8] Y. A. Carts, Diode Lasers, Nonlinear Optics, and Solid-State Lasers, 1992.

[9] B. E. A. Saleh, and M.C. Teich, Fundamentals of Photonics, A Wiley-Interscience Publication (1991).

[10] O. Svelto, Principles of Lasers, Plenum Press, New York and London, 1979. 